UDC 811.131.1'367.623

DOI 10.18485/italbg.2016.1.5

Marija Mitrović

Università di Belgrado

\title{
LA SINTASSI FORNACIARIANA IN CONFRONTO AD ALCUNE GRAMMATICHE SETTECENTESCHE ED OTTOCENTESCHE
}

\begin{abstract}
Il presente contributo propone l'analisi della Sintassi italiana dell'uso moderno di Raffaello Fornaciari nonché dei capitoli dedicati alla sintassi nelle opere di alcuni grandi grammatici italiani settecenteschi ed ottocenteschi (Soave, Corticelli, Puoti, Gigli e Romani). Gli autori precedenti al Fornaciari mostrano scarso interesse per gli argomenti sintattici trattando la sintassi alquanto diversamente dal Fornaciari. Il loro approccio alla sintassi è limitato all'analisi delle parti del discorso (concordanza, reggenza e collocazione delle parole), mentre la proposizione, nella maggioranza dei casi quella semplice, è esaminata esclusivamente nell'ambito di questi argomenti. Solo nell'opera del Romani sussiste un'analisi approfondita del periodo nonché una tassonomia ramificata affine alle tassonomie moderne. D'altra parte, l'opera del Fornaciari, ritenuta la prima moderna sintassi della lingua italiana, prende in considerazione temi sintattici precedentemente trascurati oppure studiati solo superficialmente. Oltre all'analisi dettagliata e la classificazione dei complementi, il Fornaciari, per la prima volta nella linguistica italiana, propone una chiara, precisa e sistematica classificazione delle proposizioni subordinate. Inoltre si nota una notevole differenza sul piano del metalinguaggio in quanto quello adoperato dai suoi predecessori era spesso impreciso mentre quello fornaciariano si avvicina al metalinguaggio moderno.

Parole chiave: Fornaciari, sintassi, grammaticografia, approccio alla sintassi, trattazione delle parti del discorso, trattazione delle proposizioni.
\end{abstract}

\section{INTRODUZIONE}

La Sintassi italiana dell'uso moderno di Raffaello Fornaciari (1881), "prodotto più maturo della tradizione grammaticale italiana prenovecentesca" (Patota 1993: 135), costituisce uno spartiacque essenziale nella

\footnotetext{
*m.marija3@gmail.com
} 
grammaticografia italiana in quanto, per la prima volta, gli argomenti sintattici vengono trattati indipendentemente da altri livelli linguistici, con una notevole chiarezza e in maniera del tutto nuova e moderna. Consultando le grammatiche italiane precedenti all'opera del Fornaciari, segnaliamo una dedizione decrescente alla sintassi la quale, secondo quanto afferma Giovanni Nencioni nella Prefazione alla nuova edizione della Sintassi fornaciariana, "è stata, fin dall'antichità, la parte meno coltivata della descrizione grammaticale" (Nencioni 1974: XVI) e "non riuscì da un lato a emanciparsi dall'analisi logica, dall'altro a raggiungere il livello di sintassi del periodo, restando fino all'età moderna, confinata piuttosto nell'analisi dei costrutti di parola (cioè nella morfologia, o etimologia, delle partes orationis) che nell'analisi della proposizione" (ibid). Nel presente contributo ci proponiamo di mostrare le differenze riguardanti la trattazione delle questioni sintattiche in alcuni autori principali del Sette e dell'Ottocento e nel Fornaciari nonché di mettere in evidenza la modernità del modello fornaciariano. Raffaello Fornaciari introdusse nella linguistica italiana un approccio nuovo alle questioni sintattiche, acquisito dai romanisti tedeschi, e perciò la sua opera viene considerata il primo manuale moderno di sintassi italiana. Le opere degli autori settecenteschi ed ottocenteschi (Salvatore Corticelli 1745, Francesco Soave 1771, Basilio Puoti 1833, Mariano Gigli 1818 e Giovanni Romani 1826) saranno messe a confronto a quella del Fornaciari. La particolare attenzione sarà prestata all'impostazione generale dei capitoli dedicati alla sintassi e ai rapporti tra le opere e gli autori. Si tenga presente che il periodo che affrontiamo nel presente lavoro è stato segnato da "due linee di elaborazione grammaticale - quella "alta", che potremmo ribattezzare teorico-filosofica, e quella "bassa", che potremmo definire didattico-empirica" (Patota 1993: 124). Dopo l'analisi dettagliata delle grammatiche e basandoci sulle osservazioni di Patota, possiamo concludere che le opere di Corticelli, Soave e Puoti fanno parte del cosiddetto "filone basso" in quanto propongono un modello normativo-didattico, mentre le opere di Gigli e di Romani, basate sul modello teorico-filosofico, appartengono al "filone alto".

La grammatica ragionata, di stampo normativo, scritta dal padre Francesco Soave (1817), pubblicata per la prima volta a Parma nel 1771, "costituì una vera novità nel campo degli studi sulle regole della nostra lingua" (Fornara 2004: 251). La novità dell'opera del Soave riguarda in primo luogo il titolo dal momento che "nessuno, in Italia, aveva accostato prima del Soave l'aggettivo ragionata alla parola grammatica" nonché la "modernità di impostazione e di impianto normativo" (ibid). Le opere che il Soave prese a modello per la sua grammatica, specialmente per l'intento di affrontare la lingua in maniera ragionata, furono la Grammair de Port- 
Royal e la Logique de Port-Royal (Patota 1993, Fornara 2004). Come verificheremo in seguito, Francesco Soave nella sua Grammatica rivela un notevole interesse per la sintassi la cui ricomparsa, come sostiene Fornara, insieme "all'apparire della grammatica ragionata [...] è la più importante novità che caratterizza il panorama del Settecento italiano" (Fornara 2004: 254). Stefano Telve (2002: 22) asserisce che "forti analogie stringono" il Soave e il Corticelli in quanto anche quest'ultimo nella sua opera Regole ed osservazioni della lingua toscana dedica l'ampio spazio agli argomenti sintattici, seguendo il modello della sintassi latina del gesuita portoghese Manoel Alvarez (Nencioni 1974: XVIII), ovvero alla costruzione toscana trattata nell'ambito del secondo libro della sua grammatica. Il terzo grammatico appartenente al "filone basso", la cui opera Regole elementari della lingua italiana fu pubblicata per la prima volta nel 1833 a Napoli, è il napoletano Basilio Puoti. Nencioni nella Prefazione afferma che le Regole "sono in verità il risultato, ridotto a formule didattiche, di un corso plurisecolare di trattazione grammaticale normativa, umanisticamente centrata sull'esempio contestuale, per lo più trecentesco" (Nencioni 1974: XIX). Inoltre, l'importanza del Puoti (1856) sta nel fatto che egli adopera i termini sintattici affini a quelli della linguistica moderna come mostreremo in seguito.

Come abbiamo già osservato, le opere del Gigli e del Romani fanno parte del "filone alto" e pertanto la loro impostazione è di gran lunga diversa dalle opere degli altri tre grammatici. Lo stesso titolo Lingua filosofico-universale pei dotti di Mariano Gigli (1818) suggerisce il proposito di analizzare la lingua facendo più riferimenti filosofici che linguistici. Il Gigli considera la lingua un fenomeno universale rinunciando "persino a ogni intento normativo, in favore della descrizione della lingua al solo livello teorico a partire da criteri prevalentemente semantici, senza toccare il piano sintattico e senza raggiungere i risultati nuovi e di rilievo" (Fornara 2008: 93). D'altra parte, pur appartenendo al "filone alto", la Teorica della lingua italiana di Giovanni Romani (1826) ha un'impostazione ben diversa con chiari riferimenti sia morfologici che sintattici. Esaminando dettagliatamente i fenomeni sintattici relativi alla frase semplice e al periodo, il Romani fu l'unico autore, prima del Fornaciari, a proporre una tassonomia delle frasi subordinate. Nondimeno, l'opera del Romani è colma di "numerosissime considerazioni di carattere prevalentemente semantico-lessicale, e con una tendenza esasperata alla catalogazione delle forme, distinte anch'esse in base alla loro funzione logico-semantica" (Fornara 2008: 93). 


\section{FRANCESCO SOAVE - GRAMMATICA RAGIONATA DELLA LINGUA ITALIANA}

Il capitolo dedicato alla sintassi occupa una quarantina di pagine e viene suddiviso in quattro "sezioni". Nella brevissima introduzione al capitolo il Soave (1817: 106) riporta una sintetica definizione ed etimologia del termine sintassi precisando che deriva dalle due parole greche (syn e tasso) e sottintende "coordinazione, ossia ordinata disposizione e connessione delle cose". La prima sezione riguarda la concordanza, la seconda il reggimento, mentre la terza analizza la frase ovvero la costruzione. D'altra parte, l'argomento della quarta sezione sono le figure grammaticali, materia che non viene esaminata negli odierni manuali di sintassi. Lo scopo della prima sezione sulla concordanza è di mostrare la maniera in cui l'aggettivo si accorda con il nome nonché il verbo con il soggetto di una proposizione. Trattando il reggimento nella seconda sezione, Francesco Soave individua e classifica i nomi retti da verbi intransitivi, i nomi retti da verbi transitivi e quelli retti da altre parti del discorso. Infine, fa riferimento ai verbi introdotti da altri verbi e dalle congiunzioni. Nella sezione riguardante la frase il Soave si dedica soltanto alla questione dell'ordine delle parole individuando due tipi di frasi: la costruzione semplice e quella inversa. Tale suddivisione viene basata esclusivamente sulla collocazione dei costituenti frasali: la frase con il soggetto al primo posto è definita frase semplice, mentre la frase con l'ordine delle parole diverso è definita inversa. Secondo quanto sostiene lo stesso Soave (1817: 120), la costruzione semplice "renderebbe il discorso troppo noioso" e pertanto "per dargli più grazia e leggiadria la Costruzione molte volte si varia". Bisogna accentuare che il Soave, riportando un numero rilevante di esempi, riconosce nel verbo l'elemento principale della frase idoneo ad alterare il suo senso e la sua struttura. Dedica più attenzione al numero, allo stato verbale e all'uso delle congiunzioni relative che e cui mostrando con gli esempi il modo in cui queste categorie alterano il significato frasale. Analizzando la sintassi del Soave, si conclude che tutte le sue spiegazioni sintattiche sono nella funzione dell'armonia e della chiarezza della frase e del discorso. Inoltre, come del resto negli altri autori le cui opere saranno analizzate in seguito, nel Soave l'approccio alla sintassi è basato sull'analisi del verbo e della sua posizione all'interno della frase e del discorso. Avremo occasione di notare che l'argomento basilare degli autori prefornaciariani è stato il verbo e le sue classificazioni e suddivisioni in base ai vari criteri, piuttosto semantici che sintattici. 


\section{SALVATORE CORTICELLI - REGOLE ED OSSERVAZIONI DELLA LINGUA TOSCANA}

Nell'opera di Salvatore Corticelli la sintassi viene affrontata nell'ambito del secondo capitolo intitolato "Della Costruzione Toscana" all'inizio del quale l'autore paragona i termini sintassi e costruzione osservando che "la costruzione, con Greco vocabolo chiamata sintassi, è quella conveniente disposizione, la quale debbono avere fra se le parti dell'orazione" (Corticelli 1885: 115). Segue la divisione della frase in costruzione semplice e costruzione figurata (definite anche regolare e irregolare) basata sull'ordine delle parole, analoga a quella del Soave, con un'unica differenza nel termine figurata anziché inversa. Il concetto di questa partizione è uguale a quello dell'autore analizzato nel capitolo precedente e lo provano le definizioni dello stesso Corticelli: "La costruzione semplice, o sia regolare, è quella, che segue l'ordine naturale, e le regole della Gramatica..." e avanti "La figurata è quella, che si allontana dall'ordine naturale, e dalle comuni regole della Gramatica" (ibid). Con quattordici regole riguardanti la collocazione delle parti dell'orazione il Corticelli prescrive la posizione del sostantivo nella funzione di soggetto, del verbo nella funzione di predicato nonché di altre parti del discorso quali oggetto (il caso), preposizione (il segno) e articolo. In seguito, similmente al Soave, il Corticelli si occupa in maniera assai sistematica e concisa della dipendenza e della concordanza delle parti del discorso proponendo un cospicuo numero di regole e di eccezioni. Il resto del capitolo viene dedicato alla classificazione dei verbi basata sulla reggenza verbale e "la descrizione dei verbi è assai precisa e dettagliata, tanto da far pensare ad una grammatica valenziale" (Skytte 1990: 275). Dipendentemente dai complementi il Corticelli individua i verbi attivi, assoluti, neutri, impersonali, locali.

\section{BASILIO PUOTI - REGOLE ELEMENTARI DELLA LINGUA ITALIANA}

Basilio Puoti (1856: 193) definisce la sintassi come una regolata disposizione e dipendenza tra le parole del discorso distinguendone due tipi: la sintassi semplice e la sintassi figurata. Nell'ambito della sintassi semplice individua la sintassi di concordanza e quella di reggimento offrendone un'analisi dettagliata. Un fatto tutto nuovo è la comparsa del temine proposizione e la suddivisione in proposizione semplice e proposizione composta. Il termine proposizione composta adoperato dal Puoti equivale a quello che nella linguistica moderna è conosciuto come frase complessa o periodo e la definizione riportata da questo autore ottocentesco è affine a quelle moderne: "composte sono un complesso di proposizioni semplici legate tra loro 
per modo, che tutte dipendano da una di esse, che n'è la principale" (Puoti 1856: 194). Analizzando le opere di altri linguisti e grammatici del Sette e dell'Ottocento, non abbiamo rilevato una tale descrizione della frase e i termini semplice, composta e principale, adoperati dal Puoti, costituiscono uno dei primi passi verso la moderna sintassi del periodo. Nonostante questo notevole cambiamento, si segnala ancora la mancanza di classificazioni delle frasi dipendenti che per la prima volta nella grammaticografia italiana vedrà la luce nell'opera del Fornaciari (1881). Similmente agli altri autori presi in esame, il Puoti si occupa anche della sintassi di concordanza e della sintassi di reggimento. La sintassi di concordanza riguarda l'accordo tra aggettivi e sostantivi, tra verbi e nomi e participi, mentre la sintassi di reggimento intende quello che la moderna sintassi definirebbe la valenza di sostantivi, verbi e preposizioni.

\section{GIOVANNI ROMANI - TEORICA DELLA LINGUA ITALIANA}

L'opera del Romani (1826) comprende due volumi dedicati rispettivamente alla morfologia e alla sintassi. Il volume sulla sintassi è diviso in tre sezioni dedicate alle proposizioni, alle argomentazioni e al discorso. La proposizione, sezione che ci interessa di più, è analizzata in base alle quattro caratteristiche seguenti: qualità, quantità, forma ed uso. Il modo in cui il Romani esamina la proposizione è assai diverso da quello del Fornaciari e tra le differenze principali spicca la divisione logico-semantica delle frasi. La qualità della proposizione viene definita come "varie proprietà che presentano le medesime, o rispetto all'indole assoluta o relativa degli attributi loro, oppure rispetto alle varie modificazioni della loro copula o verbo..." (Romani 1826: 153). Malgrado queste proposizioni siano denominate attributive, non possono essere intese come proposizioni attributive nelle tassonomie moderne in quanto il Romani prende in considerazione esclusivamente le proposizioni semplici basando la loro classificazione in dipendenza del rapporto tra attributo e altre parti della frase. Secondo la sua tassonomia riguardante la qualità, sussistono le assolute, relative, modali, necessarie, contingenti, possibili, impossibili e quelle identiche. Il capitolo più stimolante è quello sulla forma delle proposizioni in cui è attuata la divisione bipolare fra complete o incomplete, semplici o composte, complesse o incidenti, convertibili o opposte, pure o affette. Riteniamo che sia importante accentuare la definizione delle frasi semplici e quelle composte in quanto maggior rilievo viene dato al soggetto e all'attributo piuttosto che al predicato. Il Romani (1826: 178) definisce proposizioni semplici quelle "che non presentano che un solo soggetto reggitore ed un solo attributo", mentre individua tre tipi di proposizioni composte: quelle che hanno due o più soggetti, quelle che hanno due o più attributi e, infine, quelle "nelle 
quali lo stesso soggetto principale col suo attributo è riferito ad uno o più rapporti accidentali". Un altro argomento affrontato dal Romani, inesistente nelle altre grammatiche esaminate per il presente lavoro, è la questione della paratassi e dell'ipotassi. Il Romani distingue la composizione per aggregazione e per connessione, le quali corrisponderebbero alla coordinazione e alla subordinazione nelle grammatiche odierne. Nell'ambito della connessione, in base alla congiunzione che collega la principale e la subordinata, viene proposta una classificazione di 12 tipi di proposizioni subordinate, affine alle tassonomie moderne: condizionali, razionali o dimostrative, motivali o finali, causali, discretive, esclusive, eccettive, accrescitive o ampliative, diminutive o limitative, comparative, avversative, aggiuntive.

\section{MARIANO GIGLI - LINGUA FILOSOFICO-UNIVERSALE PEI DOTTI}

L'impostazione filosofico-semantica del Gigli (1818) è di gran lunga diversa dalle impostazioni delle altre opere esaminate finora. Secondo quanto afferma lo stesso autore, la lingua è un fenomeno universale e come tale sarà affrontato nel corso della sua opera, la quale risulta colma di spiegazioni teoriche basate sulla filosofia e priva di buoni esempi linguistici. Il Gigli (1818: 8) parte dal concetto di lingua universale ossia dalla convinzione che tutte le lingue siano simili e possano essere analizzate nella stessa maniera in quanto tutte hanno "un fondo comune; vale a dire ànno comune, ciò che forma l'assoluta essenza del linguaggio. Considerato come semplice effetto naturale". L'opera ha due parti, la prima delle quali tratta elementi del discorso ovvero la morfologia e la seconda parti del discorso ovvero la sintassi. Potremmo concludere che tutta la sintassi del Gigli, particolarmente la seconda sezione intitolata "Situazioni degli oggetti", si basa su un unico termine ed è quello di oggetto. La prima sezione è dedicata a varie determinazioni delle voci, quali determinazione degli oggetti e determinazione delle azioni, le quali alternano o completano il significato dei sostantivi e verbi. Nella seconda sezione sono analizzati vari tipi di oggetti quali oggetto cardinale, oggetto nominato, oggetto determinato, oggetto chiamato e via dicendo.

\section{RAFFAELLO FORNACIARI - SINTASSI ITALIANA DELL'USO MODERNO}

Per la prima volta nella grammaticografia italiana un'intera opera viene dedicata esclusivamente alla sintassi. Le novità introdotte dal Fornaciari (1881) sono numerosissime e si rispecchiano sia nell'impostazione 
generale dell'opera sia nelle chiare e sistematiche articolazioni sintattiche. Secondo Raffaello Fornaciari (1881: 7), i problemi principali da affrontare sono "molteplicità di maniere diverse", vale a dire molti costrutti presenti nella lingua toscana parlata, non ancora presenti nelle opere scritte, nonché la mancanza di interesse per la sintassi dei grammatici precedenti. Infatti l'autore afferma che i grammatici italiani non "hanno dato a questa materia l'estensione debita, o non hanno saputo riconoscere i veri limiti" (ibid). Nella prefazione della Sintassi il Fornaciari fa una breve rassegna delle opere dei grammatici italiani sottolineando la loro noncuranza relativa agli argomenti sintattici. Secondo il Fornaciari, quegli autori prendevano in considerazione solo le caratteristiche generali delle parti del discorso trascurando completamente il loro uso ovvero le loro funzioni. In quanto gli utenti delle loro grammatiche erano i madrelingua italiani, ritenevano superfluo analizzare minuziosamente la sintassi delle parti del discorso. Inoltre, il Fornaciari accentua il fatto che le sintassi precedenti alla sua opera fossero affini ai dizionari, vale a dire contenessero spiegazioni dettagliate riguardo ai diversi usi delle parti del discorso, particolarmente, dei verbi. Dal momento che la sintassi veniva trascurata dai suoi predecessori, il Fornaciari seguì l'esempio dei romanisti tedeschi, tra cui il più importante 'l'illustre Diez, che con sì bell'ordine e con tanta esattezza confrontò le forme sintattiche delle lingue romanze" (ibid). Il Fornaciari acquisì dai grammatici tedeschi, che riteneva "più diligenti e più metodici" (ibid) dei grammatici italiani, l'accuratezza e la chiarezza di esposizione dei problemi sintattici. Oltre a Federico Diez, il Fornaciari prese a modello il Vockeradt, altro romanista tedesco, e la sua Grammatica italiana, pubblicata a Berlino nel 1878, nella quale "raccoglie e ordina, si può dir, tutte le varietà dei costrutti italiani, e somministra una larga copia d'esempi appropriati, sì antichi come moderni" (Fornaciari 1881: 7).

L'impostazione generale e la terminologia della Sintassi italiana dell'uso moderno sono affini a quelle delle moderne sintassi italiana. L'opera è divisa in tre capitoli principali i quali trattano, rispettivamente, le parti del discorso, la proposizione e la collocazione delle parole. Nel primo capitolo, che la linguistica moderna definirebbe la morfosintassi, troviamo le informazioni riguardanti la morfologia delle parole con qualche riferimento alla sintassi ovvero al loro uso nell'ambito della frase. Il Fornaciari ai suoi lettori offre una vasta e dettagliata suddivisione e classificazione (con tutti i casi particolari e tutte le eccezioni) di sostantivi, aggettivi, numeri, pronomi, verbi, avverbi, preposizioni e congiunzioni. Nel secondo capitolo della sua Sintassi, consistente di cinque sezioni trattanti rispettivamente i costituenti frasali (soggetto, predicato e oggetto), i complementi, le proposizioni subordinate, la forma della proposizione in generale, i modi e i tempi nella proposizioni subordinate e coordinamento delle proposizioni, 
il Fornaciari propone, per la prima volta, una chiara e approfondita sintassi del periodo. Le proposizioni subordinate in primo luogo vengono divise "collo stesso ordine de' complementi" (Fornaciari 1881: 8) in attributive, soggettive, oggettive e avverbiali, le quali, a loro volta, vengono suddivise in altri nove tipi (locali, temporali, causali, finali, condizionali, concessive, di maniera e guisa, comparative e consecutive). Bisognerebbe sottolineare che il Fornaciari nell'ambito della sintassi della proposizione non trascura gli argomenti quali dislocazione a destra e dislocazione a sinistra, denominate da lui duplicazione dell 'oggetto, modo esplicito ed implicito, nonché la forma affermativa, negativa e interrogativa della frase. Il Fornaciari conclude la sua opera sulla sintassi della lingua italiana con il capitolo dedicato alla collocazione delle parole in cui propone l'ordine delle parti del discorso (articoli, aggettivi e sostantivi, numerali e sostantivi...), degli elementi della proposizione (soggetto e predicato, predicato nominale, oggetto...), delle proposizioni subordinate e coordinate e, infine, dei segni d'interpunzione.

\section{CONCLUSIONI}

Confrontando i maggiori autori settecenteschi ed ottocenteschi e l'opera di Raffaello Fornaciari, si nota la carenza di mature argomentazioni sintattiche nelle opere prefornaciariane. La presente analisi ha mostrato che la sintassi degli altri autori si basava prevalentemente sulla concordanza delle parti del discorso. L'analisi della sintassi del periodo risulta assai modesta con la terminologia insufficientemente sviluppata e, a volte, poco chiara. Il Soave, il Corticelli e il Puoti sono stretti dai fortissimi legami rispecchiati per lo più nell'impostazione generale dell'opera nonché nel simile approccio agli argomenti sintattici. La loro sintassi si impernia sugli stessi argomenti quali accordo tra gli elementi della frase, reggenza e collocazione delle parti del discorso mentre l'approccio con cui esaminano la proposizione è di gran lunga difforme da quello fornaciariano ovvero quello odierno. La sintassi della proposizione è limitata alla collocazione delle parti del discorso e l'unica suddivisione delle proposizioni viene basata sull'ordine regolare o irregolare dei costituenti frasali ovvero sulla disposizione naturale o quella marcata. L'unica eccezione, riguardo alla sintassi della proposizione, è costituita dall'opera di Giovanni Romani (1826) la quale, pur appartenendo al periodo prefornaciariano, propone una ramificata classificazione delle frasi subordinate nonché un consistente studio di vari problemi sintattici. L'opera del Romani, in quanto comprende sia gli argomenti di concordanza, collocazione e figure grammaticali che la dettagliata tassonomia delle proposizioni subordinate, potrebbe essere considerata un passaggio tra l'epoca prefornaciariana e quella fornaciariana. D'altra parte, Raffaello For- 
naciari, con le due opere quasi rivoluzionarie ${ }^{1}$, ha posto le fondamenta della moderna linguistica italiana e la sua modernità "emerge prepotentemente proprio nella Sintassi, che viene affrontata e discussa in modo nuovo, più completo e organico, coinvolgendo anche aspetti ancora poco o per nulla indagati dalla nostra grammaticografia: egli non si ferma infatti all'uso e alla concordanza delle singole parti del discorso - argomento che abbiamo già incontrato nei pochi grammatici che si interessarono, prima di lui, a questioni sintattiche - ma estende l'indagine alla sintassi e all'analisi della proposizione e del periodo, fornendo anche una classificazione delle proposizioni" (Fornara 2008: 102). La Skytte (1990: 276) afferma che "ancora oggi si può consultare Fornaciari con profitto", precisando che, "se ciò è vero, non è tanto merito del Fornaciari quanto del carattere di stasi della grammatica italiana del '900, almeno fino agli anni settanta. L'orientamento prevalentemente filologico della grammaticografia italiana è stato ulteriormente accentuato per via del forte influsso del pensiero crociano" (ibid). Le differenze tra il Fornaciari e gli altri autori presi in esame non riguardano solamente la sintassi del periodo, ma anche il trattamento di tutte le altre questioni sintattiche: è completamente cambiato l'approccio alla sintassi. Mentre le grammatiche prefornaciariane sono basate sull'analisi delle parti del discorso, per la prima volta nell'opera fornaciariana emerge uno studio approfondito delle funzioni delle parti del discorso, definite parti secondarie della proposizione. Per quanto riguarda i complementi, a differenza dei suoi predecessori, egli propone una dettagliata suddivisione semantica alquanto simile a quelle presenti nei moderni manuali di analisi logica.

Il metalinguaggio presente nell'opera fornaciariana costituisce un altro cambiamento rispetto al passato ovvero un altro passo verso la linguistica moderna. Gli autori sette e ottocenteschi adoperano terminologia piuttosto antiquata: nell'opera del Corticelli, ad esempio, troviamo il caso invece dell'oggetto oppure il segno invece della preposizione. Similmente, sia il Soave che il Corticelli adoperano il termine la costruzione invece di proposizione mentre il Romani scrive della composizione per aggregazione e per connessione invece della coordinazione e subordinazione. Di frequente, le differenze nel linguaggio metalinguistico sono dovute al fatto che i grammatici prefornaciariani usassero i criteri diversi da quelli moderni nella suddivisione e classificazione di fenomeni sintattici in modo che nel Romani sussistano i termini quali le proposizioni necessarie e contingenti, le proposizioni possibili e impossibili, le proposizioni discretamente quantitative, le proposizioni composte e convertibili e via dicendo. Sia il Soave che il Corticelli e il Romani affrontano accuratamente l'ordine degli elementi

${ }^{1}$ Oltre alla Sintassi dell'uso moderno, Raffaello Fornaciari scrisse Grammatica dell'uso moderno (1879). 
della frase usando i termini la costruzione semplice e la costruzione figurata per indicare rispettivamente la frase con la disposizione di elementi naturale (ovvero l'ordine non marcato) e quella con la disposizione non naturale (ovvero l'ordine marcato). D'altra parte, la terminologia adoperata dal Fornaciari è conforme alla terminologia metalinguistica moderna, benché notiamo la mancanza di alcuni concetti moderni "quali, ad es., quelli di frase nominale, di aspetto verbale, di segmentazione, di nominalizzazione, di sequenza progressiva o regressiva, di presupposizione, di distribuzione, di distinzione fra struttura superficiale e struttura profonda" (Nencioni 1974: XXVI). Quanto all'opera di Mariano Gigli (1818), possiamo constatare che essa rappresenta un esempio particolare tra le opere esaminate. La grammatica del Gigli è l'unica a non potersi accostare né alle opere prefornaciariane né al Fornaciari in quanto l'impostazione teorico-filosofica, l'approccio universale alla lingua, gli argomenti e, soprattutto, il metalinguaggio sono di gran lunga difformi dagli altri autori.

Vista l'importanza dell'opera fornaciariana, caso a parte nella lunga tradizione linguistica italiana, e le sue differenze riguardo alle opere precedenti, potremmo concludere che sarebbe necessario paragonarla anche alle grammatiche successive, quelle del Novecento o anche del Duemila, per esaminare la loro complementarità al Fornaciari o il loro distacco da lui.

\section{BIBLIOGRAFIA}

Corticelli, S. (1885). Regole ed osservazioni della lingua toscana ( $1^{\mathrm{a}} \mathrm{ed}$. 1745). Napoli: Dalla tipografia di Gabriele Gentile.

Fornaciari, R. (1881). Sintassi italiana dell'uso moderno. Firenze: Sansoni. Fornaciari, R. (1882). Grammatica italiana dell'uso moderno. Firenze: Sansoni.

Fornara, S. (2004). Francesco Soave e la grammatica del Settecento. In C. Marazzini \& S. Fornara (a cura di), La Grammatica ragionata di Francesco Soave tra pregiudizi, tradizione e modernità (pp. 251-260). Alessandria: Edizione dell'Orso.

Fornara, S. (2008). Breve storia della grammatica italiana. Roma: Carocci. Gigli, M. (1818). Lingua filosofico-universale pei dotti. Milano: Società tipografica de' Classici italiani.

Nencioni, G. (1974). Presentazione. In R. Fornaciari, Sintassi italiana dell'uso moderno (pp. V-XXVIII). Firenze: Sansoni.

Patota, G. (1993). Storia della lingua italiana, 1. I luoghi della codificazione. In L. Serianni \& P. Trifone (a cura di), I percorsi grammaticali (pp. 93-137). Torino: Einaudi. 
Puoti, B. (1856). Regole elementari della lingua italiana (1 $1^{\mathrm{a}}$ ed. 1833). Pisa: Fratelli Nistri.

Romani, G. (1826). Teorica della lingua italiana. Milano: Per Giovanni Silvestri.

Skytte, G. (1990). Dall'Alberti al Fornaciari. Formazione della grammatica italiana. Revue Romana, 25, 2, 268-278.

Soave, F. (1817). Gramatica ragionata della lingua italiana ( $1^{\text {a }}$ ed. 1771). Milano: Società tipografica de'classici italiani.

\author{
RAFFAELLO FORNACIARI'S SYNTAX IN COMPARISON \\ TO SOME GRAMMARS OF EIGHTEENTH AND NINETEENTH CENTURY
}

\title{
Summary
}

The purpose of this paper is to analyze the Sintassi italiana dell'uso moderno of Raffaello Fornaciari and chapters treating the syntax in the works of some great Italian grammarians of eighteenth and nineteenth century (Soave, Corticelli, Puoti, Gigli and Romani). The authors prior to Fornaciari demonstrate the lack of interest in the syntactic arguments dealing with the syntax quite differently from Fornaciari or contemporary authors. The syntax of all five above-mentioned authors is based on the analysis of parts of speech, precisely, on the concordance, regency and collocation of words whereas the proposition, in most cases the simple one, was treated within these topics. Solely, in the work of Romani exists serious analysis of compound sentence. On the other hand, the Fornaciari's work, considered as the first modern syntax of Italian language, treats syntactic issues previously neglected or insufficiently studied. Besides detailed analyses and classification of complements, Fornaciari, for the first time in the Italian linguistics, proposes a clear, precise and systematic classification of the subordinate clauses. The significant difference is noticed in the term of the metalanguage inasmuch as the one used by elder grammarians was, often, uniform and inaccurate whereas the Fornaciari's one corresponds to the modern metalanguage.

Keywords: Fornaciari, syntax, grammaticography, approach to syntax, approach to parts of speech, approach to propositions. 